
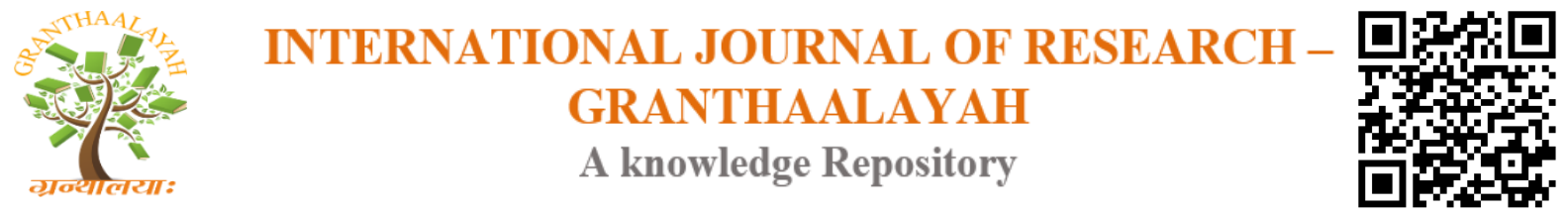

Science

\title{
THE CAUSAL LINK BETWEEN VACCINATION ADJUVANTS AND AUTISM
}

\author{
Thomas Prevenslik *1 \\ ${ }^{* 1}$ Researcher, QED Radiations, Berlin, Germany
}

\begin{abstract}
Adjuvants essential in vaccines to stimulate activation of the immune system comprise submicron $(<100 \mathrm{~nm}$ ) nanoparticles (NPs) of aluminum hydroxide that upon vaccine injection cross the blood-brain-barrier $(\mathrm{BBB})$ and because of their low solubility may remain in the brain for an extended time. Despite over a century of use, the mechanism underlying the stimulation of the immune system by adjuvants has not been understood. Solar UV can optically stimulate most immune systems in the skin, but solar UV is not available inside the brain. By the process of simple QED, the NPs lacking heat capacity by the Planck law conserve heat from brain tissue by emitting UV radiation instead of increasing in temperature. Hence, the causal link between vaccination adjuvants and autism is UV stimulation of the immune system from constituent NPs that cross the BBB. But the UV also damages the DNA of brain tissue and increases the pro-inflammatory interleukin (IL-6) cytokine levels common in

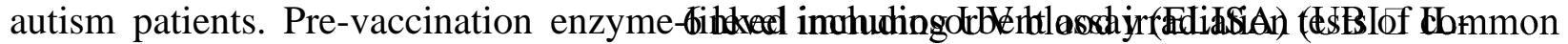
in immune-modulating therapy are suggested to determine if a patient will have an adverse vaccination response. Patients having high IL-6 levels are not recommended for vaccination.
\end{abstract}

Keywords: Adjuvants; Autism; Nanoparticles; Planck Law; Quantum Mechanics; UV; Vaccinations.

Cite This Article: Thomas Prevenslik. (2019). "THE CAUSAL LINK BETWEEN VACCINATION ADJUVANTS AND AUTISM." International Journal of Research Granthaalayah, 7(6), 288-295. https://doi.org/10.29121/granthaalayah.v7.i6.2019.805.

\section{Introduction}

Autism like Parkinson's and Alzheimer's have unknown origins and involve genetic and environmental factors. Genetic factors are inherited. Environmental factors are acquired including adjuvants of $<100 \mathrm{~nm}$ NPs in vaccines to stimulate activation of immune system antigens, the adjuvants approved by the FDA about a century ago. Absent adjuvants, stimulation of the immune system would be ineffective. The FDA then as now considers adjuvants safe, but tests to verify safety are still incomplete. 


\section{Nanoparticles}

Micrographs show NPs in aluminum adjuvants agglomerate into clusters of about 2-20 microns as shown in Fig. 1. Scale bar $=2$ microns. DNA damage from $>1$ micron clusters is insignificant. However, DNA damage from the NPs located at the cluster periphery is significant, but mostly ignored by the FDA.

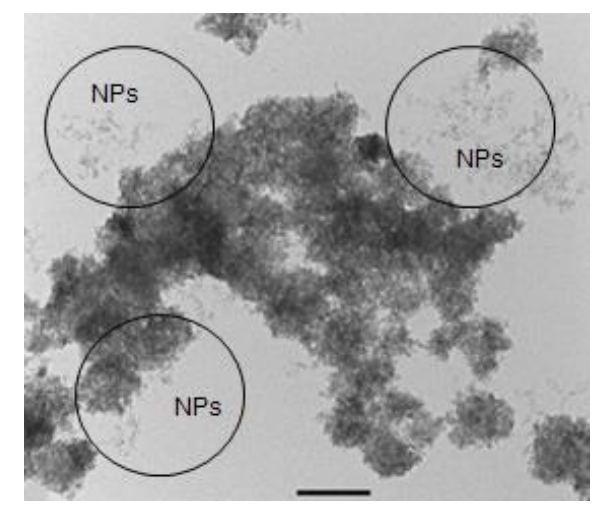

Figure 1: NPs in Aluminum Adjuvants

\section{DNA Damage}

Autism aside, nanotechnology experiments for more than 2 decades show adding NPs to biological fluids damage DNA. In 2003, NPs correlated with reactive oxygen species (ROS), e.g., OH radicals and the $\mathrm{O}$ super oxides [1,2]. Oxidative stress was thought caused by an imbalance between damaging ROS oxidants and protective antioxidants vitamin $\mathrm{C}$ and glutathione which can damage DNA leading to loss of cell function. At that time, ROS were thought caused by NP high surface-to-volume ratios providing the link to oxidative stress and diseases including cancer, asthma, and neurological disorders.

In 2008, NP driven DNA damage from ROS were found [3] to induce DNA damage in A549 cells treated with urban dust. Comet assays showed single and double-strand breakage of DNA. Activation of p53 proteins related to DNA damage mimicked known ionizing EM irradiationrelated carcinogenesis pathways suggesting the ROS are created by NPs producing EM radiation, but an EM source was not identified.

Further, tests in 2008 showed [4] photodynamic therapy (PDT) could treat cancer tumors by IR laser heating of Au NPs previously attached to the tumor surface. PDT relies on the selective uptake and retention of the NPs by the tumor. Tumor necrosis was interpreted as the effect of high temperatures from IR heating of NPs. However, DNA damage tests as for urban dust [3] were not performed in PDT because it was implicitly assumed tumor necrosis was caused as the heated NPs increased in temperature, i.e., ionizing radiation was not considered as the NPs were heated by benign IR radiation. Since then, DNA damage from NP induced EM radiation [5] and the extension to cancer [6] from NPs in genetically modified (GM) food were based on the physical argument that NPs emit EM radiation as the Planck law of quantum mechanics precludes the conservation of heat by an increase in temperature as the heat capacity of constituent atoms vanishes. 
In 2016, PDT with Ag instead of Au NPs showed [7] tumor necrosis in A549 lung cancer cells. Unlike prior PDT research, the significance was comet assays showed DNA damage after PDT. The DNA damage was not correlated with ionizing EM emission from NPs, but rather by the generation of ROS from membrane potentials suggesting mitochondria generate ROS by IR light absorption. Only then did would the mitochondrial membrane potential initiate the sequential series of chain reactions that led to tumor necrosis by ROS.

The notion of the mitochondria [8] generating ROS evolved from Mitchell's [9] theory of ATP synthesis based on a proton $\mathrm{H}+$ gradient across the inner mitochondrial membrane by hydrolysis using a complex chain of sequential redox reactions, the electron transfers from donors to acceptors assisted by enzymes. However, the mitochondria like NPs comprise nanoscale features that produce EM radiation in the UV that mediates [10] the direct production of ATP from ADP by a dehydration reaction thereby avoiding the complex and questionable sequential chain reactions in hydrolysis. Since some of ATP produced bind to cristae surfaces, standing UV is created in the nanoscopic space between adjacent cristae, i.e., the UV creates the mitochondrial potential and ROS are inconsequential. In mitochondria, UV radiation from nanoscopic features damage DNA.

NPs in nanotechnology are more common than NPs $\mathrm{n}$ biological applications. Translation of nanotechnology to medical science suggests labeling products having NPs would at least allow consumers to make a decision on purchasing the product. NPs linked to health problems are shown in Figure 2.

\section{NANOTECHNOLOGY NEEDS LABELLING}

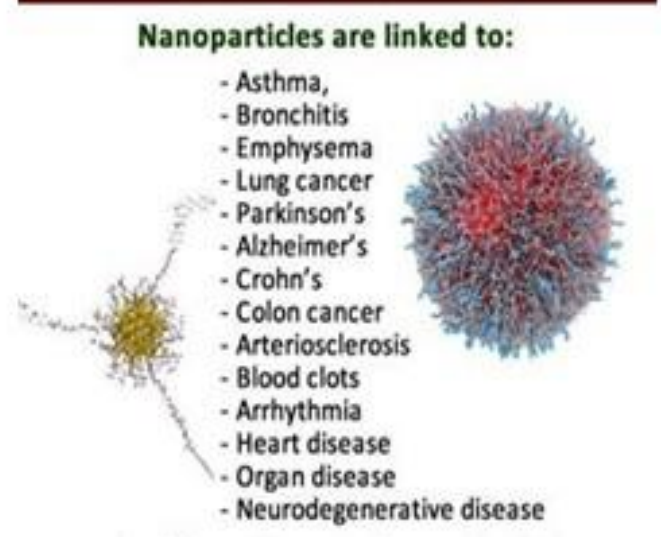

Figure 2: NP labelling in Nanotechnology

The diversity of human health problems suggests NP induced DNA damage randomly scrambles immune system genes. EM radiation in the UV is a fundamental mechanism of DNA damage that could explain gene modification in DNA, i.e., neurodegenerative like autism are likely to find origin in UV induced DNA damage in the human brain. Sunlight provides exogenous UV, but UVC at $254 \mathrm{~nm}$ is absorbed in the ozone layer and only UVB ( $280-320 \mathrm{~nm}$ ) reach the Earth to damage DNA in human skin. Regardless, solar UV is unlikely to enter the brain. An endogenous $\mathrm{UV}$ source in the brain is required, but none is known. 


\section{Proposal and Contribution}

In vaccinations, adjuvants of aluminum NPs that bypass the skin and directly enter the blood stream crossing the BBB are proposed to provide a source of endogenous UV in the brain. Unlike skin exposed to UVB, the far more damaging UVC is produced by NPs in the brain. The UV frequencies that initiate specific health problems is beyond the scope of this paper. Only the basic theory of NP induced DNA damage is presented that may lead to neurological diseases such as autism.

The contribution of the instant paper suggests the causal link between vaccinations and autism is NP induced DNA damage of the immune system by adjuvants. But the DNA damage is not important if repaired by the immune system thereby justifying the immunization of the antigen to invoke greater immune system response. Vaccinations of a great majority of patients are successful because their immune systems promptly repairs NP induced DNA damage. However, patients having weaker immune systems risk continued DNA damage that may lead to autism. To provide a means of quantifying the risk to the patient, pre-vaccination testing for IL-6 by ELISA and UBI is proposed. IL-6 is an pro-inflammatory cytokine that is enhanced by UV radiation. If IL-6 levels are high, the vaccinations are not recommended.

\section{Materials and Methods}

The causal link between vaccinations and autism depends on simple QED - a new method of analysis applicable to nanoscale heat transfer. But simple QED is not the complex light and matter interaction advanced by Feynman and others. Indeed, simple QED is readily understood by the Planck law of quantum mechanics that requires the heat capacity of constituent atoms in NPs to vanish under EM confinement. In contrast, classical physics always assumes the atom has heat capacity that produces an increase in temperature upon the absorption of heat. Simple QED differs as the NP absent heat capacity is conserved by the creation of standing EM radiation inside and across the diameter of the NP as shown in Figure 3.

\section{Simple QED}

Simple QED is based on the Planck law that precludes conservation of heat $Q$ in NPs by an increase in temperature

and

the EM confinement of standing wave in the NP by the heat $Q$ deposited in the NP surface because of high $S / V$ ratios of NPS

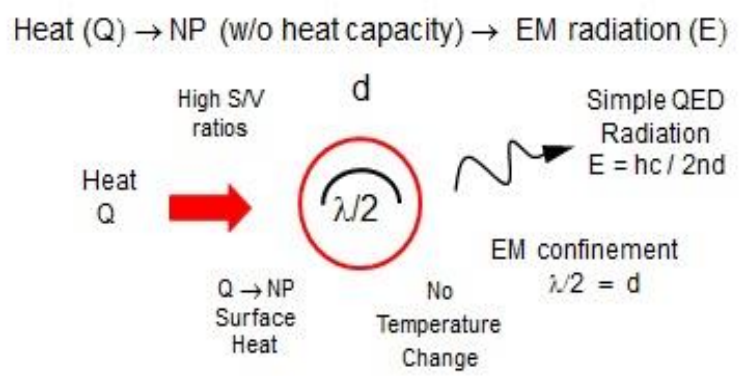

Figure 3: Simple QED conversion of heat to EM radiation 


\section{Statistical Analysis}

The causal link between vaccinations and autism is an exploratory study of how simple QED in adjuvant NPs damage DNA. Simple QED is self-evident physics providing understandable explanations [5,6,10] of experimental data from nanotechnology. Statistical analysis is not relevant.

\section{Results}

Simple QED predicts aluminum hydroxide NPs of adjuvants that enter the brain through the BBB produce size dependent EM radiation. Fig. 4 shows UVC radiation is produced for NP diameters $\mathrm{d} \sim 80 \mathrm{~nm}$.

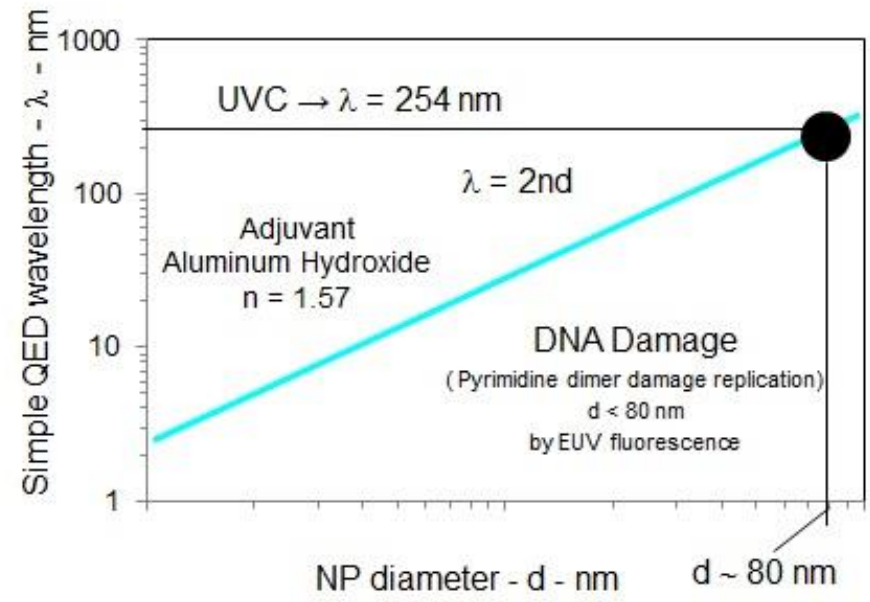

Figure 4: DNA damage from aluminum hydroxide adjuvants

UVC in the brain is significant as the pyrimidine dimer mediating replication is damaged. For NPs $>80 \mathrm{~nm}$, the EM radiation is in the UVB and also damages the DNA, but far less than the UVC. Larger NPs produce benign VIS and IR. But NPs $<80 \mathrm{~nm}$ produce EM radiation $>$ UVC. NPs $<5$ $\mathrm{nm}$ produce EM radiation $>80 \mathrm{eV}$ which is in the extreme ultraviolet (EUV). Toxicity of DNA to EUV is not known. However, the EUV also produces UVC and UVB by fluorescence, but at a low efficiency $<1 \%$.

\section{Discussion}

\section{Theories of Adjuvants and Autism}

Theories of how aluminum adjuvants cause autism are shown in Fig. 5. Current theory [11] assumes aluminum adjuvants in vaccines help the antigen in the vaccine to work more effectively, although the mechanism for immune activation is not given. Upon vaccine injection, the adjuvant in macrophages moves across the BBB into the brain. IL-6 is produced from adjuvants that in the brain that over long periods leads to autism. In the proposed theory, simple QED converts heat from the brain tissue into UV radiation that activates the immune system to provide the response for the antigen included in the vaccine. But the UV may also induce DNA damage in brain that 
enhances IL-6 levels [12,13] and increases pro-inflammatory response, thereby scrambling genes of the DNA immune system. Unlike dietary aluminum which will usually clear rapidly from the body, NPs of the aluminum adjuvants in vaccines are not soluble in body fluids and accumulate [14] in the brain. Although the immune system repairs DNA damage, the NPs continually produce UV and upon overwhelming the immune system, leads to autism.

\title{
Current theory
}

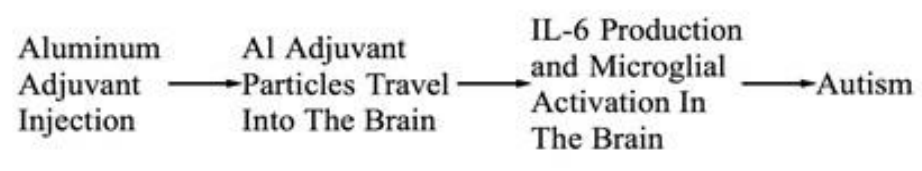

\section{Proposed theory}

\begin{abstract}
Autism is caused as simple QED induces NPS of aluminum in adjuvants to produce UV radiation that activates the antigen but also the inflammatory Interleukin IL-6 cytokine. Autism depends on the outcome of the balance between antigen activation and DNA damage determined by the immune system.
\end{abstract}

Figure 5: Current and Proposed Theories

\section{Pre-Vaccination Tests}

In theory, pre-vaccination tests would minimize the risk of autism if a suitable biomarker is identified. Autism patients are known [15] to have high levels of IL-6 which in combination with levels of aluminum in the brain suggest an immune activation mechanism produces IL-6. The secretion of IL-6 is known [16] to spontaneously increase upon exposure to UVB light. Mononuclear cells from autistic patients produced IL-6 spontaneously, while those from normal donors did not. In the simple QED production of UV from NPs in adjuvants, an increase in IL-6 levels would be expected upon vaccination. Pre-vaccination ELISA tests for high IL-6 levels in newborns would quantify a basis for allowing patients to be vaccinated to avoid NP induced autism. Vaccinations should not be given to anyone having high IL-6 levels.

In the alternative to ELISA, the simple QED induced UV source in the blood may be an suitable biomarker of autism. Ultraviolet blood irradiation (UBI) commonly [17] used in the 1950's as an immune-modulating therapy may be an acceptable biomarker. Low UBI doses kill microorganisms in the blood by damaging their DNA, while any DNA damage in host cells can be rapidly repaired by DNA repair enzymes. UBI tends to stimulate the immune system and may mediate $[18,19]$ the immune system response as UV can reverse cytokine production and block cytokine release. Compared to the IL-6 biomarker, UBI requires considerable more work to be justified as a clinical biomarker of autism.

\section{Conclusions}

DNA damage to the immune system as the causal link between vaccination adjuvants and autism is based on UV radiation emitted from NPs. But the UV radiation also activates antigens in the immune system that enhance the effectiveness of the vaccination. DNA damage and activation of the immune system is delicately balanced provided the immune system repair is not overwhelmed 
by UV induced DNA damage, otherwise continued DNA damage of the impaired immune system may lead to autism.

UV emission from NPs is a consequence of the Planck law that denies constituent atoms in NPs the heat capacity to conserve heat in brain tissue by an increase in temperature, and instead conservation proceeds by simple QED creating non-thermal standing EM waves confined within the NP. The EM confinement of the NP is caused by inherent high S/V ratios that deposit heat into the NP surface. Once the surface heat is depleted in forming the standing wave, the EM confinement vanishes and the EM wave is free to move into surrounding body tissue to damage the DNA of the immune system.

To avoid autism, it is important to know the level of DNA damage in the brain before having a vaccination containing adjuvant NPs as the induced UV will increase the DNA damage level. Prevaccination tests of autism biomarkers reviewed were the pro-inflammatory IL-6 cytokine as measured by ELISA and UBI. However, the limited time allotted in this review suggests there can be no conclusion as to a recommended pre-vaccination test for brain damage leading to autism. Suggestions for autism tests that can the measure the DNA damage level in the brain are solicited.

\section{References}

[1] Donaldson K, Stone V. 2003 Current hypotheses on the mechanisms of toxicity of ultrafine particles. Ann Ist Super Sanita ; 39: 405-410.

[2] Donaldson K. 2003; The biological effect of coarse and fine particulate matter. Occu Environ Med; $60: 313-314$.

[3] Mroz RM, Schins RPF, Li H, Jimenez LA, Drost EM, et al. 2008; Nanoparticle-driven DNA damage mimics irradiation-related carcinogenesis pathways. 2008; Eur Respir J. ; 31: 241-251.

[4] Cheng Y, Samia AC, Meyers JD, Panagopoulos I, Fei B, Burda C. 2008; Highly Efficient Drug Delivery with Gold Nanoparticle Vectors for in Vivo Photodynamic Therapy of Cancer. 2008; J. Am. Chem Soc. 130:10643-10647

[5] Prevenslik T. 2010; DNA damage by Nanoparticles. Proceedings of ASME2010 First Global Conference on Nano Engineering for Medicine and Biology, NEMB2010, Houston, TX, USA, February 7-10.

[6] Prevenslik T. 2017; Cancer by UV radiation from nanoparticles in GM food. Integr. Cancer Sci. Therap.; 4(1): 2-4.

[7] El-Hussein A. 2016; Study DNA Damage after Photodynamic Therapy using Silver Nanoparticles with A549 cell line. J Nanomed. Nanotechnol Eur Respir J.; 7:1

[8] O'Rourke B. 2007; Mitochondrial ion channels. Annual Review of Physiology; 69: 19-49.

[9] Mitchell, P. 1961; Coupling of phosphorylation to electron and hydrogen transfer by a chemiosmotic type of mechanism. Nature; 191: 144-148.

[10] Prevenslik T. 2019; ATP by Endogenous UV Radiation. www.nanoqed.org, 2019.

[11] Shaw CA, Tomljenovic L. 2013; Aluminum in the central nervous system (CNS): toxicity in humans and animals, vaccine adjuvants, and autoimmunity. Immunol Res; 56: 304-316.

[12] Kulms D, Poppelmann B, Schwarz T. 2000; Ultraviolet Radiation-induced Interleukin 6 Release in HeLa Cells Is Mediated via Membrane Events in a DNA Damage-independent Way. J Biological Chem; 20: 15060-15066.

[13] Pelton BK, Hylton W, Denman AM. 1992; Activation of IL-6 production by UV irradiation of blood mononuclear cells from patients with systemic lupus erythematosus. Clin. Exp. Immunol; 89: 251-254. 
[14] Mold M, Umar D, King A, Exley C. 2018; Aluminium in brain tissue in autism. J. Trace Elements Medicine Biology; 46: 76-82.

[15] Yang CJ, Liu CL, Sang B, Zhu XM, Du XJ. 2015; The Combined Role of Serotonin and Interleukin-6 as Biomarker for Autism. Neuroscience; 284: 290-296.

[16] Wei LW. 2011; IL-6 is increased in the cerebellum of autistic brain and alters neural cell adhesion, migration and synaptic formation. J. Neuroinflammation; 8: 52-55.

[17] Wu X, Hu X, Hamblin MR. 2016; Ultraviolet blood irradiation: Is it time to remember "the cure that time forgot". J Photochem Photobiol B.; 157: 89-96.

[18] Deeg HJ. 1988; Ultraviolet irradiation in transplantation biology. Manipulation of immunity and immunogenicity. Transplantation.; 45:845-851.

[19] Arlett CF, Lowe JE, Harcourt SA, Waugh AP, Cole J, Roza L, Diffey BL, Mori T, Nikaido O, Green MH. 1993; Hypersensitivity of human lymphocytes to UV-B and solar irradiation. Cancer research; 53: 609-614. 